\title{
A model for the life expectancy of railway switches and crossings for maintenance and renewal planning in asset management systems
}

\author{
W.-J. Zwanenburg \\ EPFL: École Polytechnique Fédérale de Lausanne, Switzerland \\ LITEP: Laboratory for Intermodality and Transport Planning, \\ Switzerland
}

\begin{abstract}
With the help of databases of the Swiss Federal Railways (SBB CFF FFS), statistical analyses are carried out on the lifetime of railway switches (points) and crossings. The expected lifetimes are attributed to different parameters which influence the speed of geometrical degradation or wear of the material, e.g. total train loads (expressed in cumulative tonnages), axle loads, train type, the quality of the foundation.

First results confirm long known basic knowledge, e.g. a gradual increase in the average replacement age due to better components and changing maintenance politics.

At the same time unexpected results are found. Examples of these are the not statistically confirmed reduced lifetime of a switch being placed in a curve, or the complete irrelevance of the switch angle on their expected life duration, although a shorter life with larger angles would be expected due to higher dynamic lateral loads and jerk. Some explanations for these unexpected results are given and are probably related to both the situation in the field and non technical issues, like maintenance policy.

With this knowledge on degradation, a model is composed which is used to forecast maintenance and renewal needs of switches and crossings To test the model, an old asset database with switches and crossings is used, and the results of the model (proposed replacements including the time of replacement) is compared with the actually carried out works.
\end{abstract}

Keywords: railways, switches and crossings, renewal, maintenance, asset management systems. 


\section{Introduction}

Railway switches and crossings (S\&C) are the devices which allow trains:

- to change tracks to continue their way in different directions;

- to change tracks to continue their way in the same direction but on different tracks;

- to cross other tracks.

Since the railway is a guided way (i.e. the track determines where the train will go, opposite to for example an automobile or a pedestrian) with only one degree of liberty (i.e. forward or backwards), S\&C are crucial to uphold the geographical advantage of modern railways: the relative and absolute small amount of space needed - compared to motorways or waterways - to transport the same amount of passengers or freight. This efficiency can only be achieved by concentrating trains from different origins with the same destination at a certain point, or vice versa. It thus also reduces the actual track length needed, because trains can be concentrated on a smaller amount of tracks.

Another important contribution of S\&C is the possibility they introduce, to divert trains to other tracks if the track which they were meant to use is blocked, for whatever reason. In this case the timetable can be upheld and the passengers or freight can still arrive at their destination, even though the original track is not available.

In other words, $\mathrm{S} \& \mathrm{C}$ form an important asset and although expressed as a part of the network length, they do not count for much, the part for S\&C of the annual amount of money used for the maintenance and renewal of all railway tracks is significant. The proper attribution of this budget is a challenging task when taking into account the size of the network, the need for big expensive machines to carry out the work at site, the often difficult access to these sites, the availability of machines and personnel and above all - on saturated or close-tosaturation networks - the availability of time to carry out maintenance (possessions).

The challenging task of proper maintenance and renewal budget attribution has lead to the development of several maintenance management tools, e.g. Decision Support Systems like RailSys or EcoTrack, as described in Rivier [2] to help the maintenance planner with his task of planning maintenance and renewal works and also to foresee future needs. The result is visible as a large rationalisation of information collection and processing (e.g. site visits, use of the measuring trains) and the maintenance and renewal planning, on networks where these tools are in use, during the last 10-20 years. This, however, only counts for plain track, i.e. straights and curves, and tunnels, bridges, signalling and overhead electrical equipment, not for S\&C.

Different reasons are identified for this lack in Jovanović and Zwanenburg [1]. The main reason is that, while for plain track, for different geometrical degradation modes (e.g. cant, twist, level, gauge) and component deterioration (e.g. cracks, gauge corner cracking, head checks, corrugation) the relation with track parameters (e.g. sub-soil quality, type of sleepers) and train load (e.g. 
number and amount of trains, axle loads) is known, as proven by Veit [3], the knowledge on degradation and deterioration processes of $\mathrm{S} \& \mathrm{C}$ has remained rudimentary. It is the knowledge on these degradation and deterioration processes which is necessary for the development of the maintenance management tools and the obtaining them is the objective of the broad study.

This article will present some results on factors determining the renewal rate of standard turnout.

\section{Degradation and wear}

At a certain moment the degradation or wear of track or switches and crossings $(\mathrm{S} \& C)$ reaches a limit on which comfortable and/or even safe train traffic cannot be guaranteed. A maintenance or renewal action is then necessary. The moment at which this limit is reached strongly depends on the train load. A heavily loaded track will show more wear than a track with only a small amount of (light) trains, unless, however, the track structure is prepared for higher loads, then this effect might be reduced.

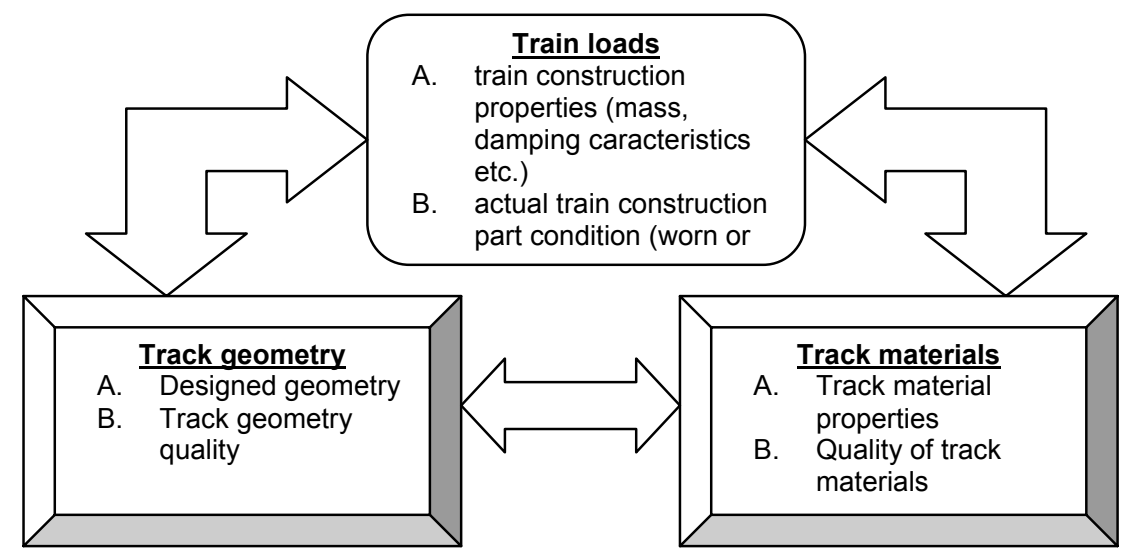

Figure 1: Relationships between the train loads, track (state of the) track materials and track geometry (quality).

As mentioned in the introduction above, two different reductions of track quality can be described: geometrical degradation and component deterioration (tear and wear). When examining the increase in degradation or wear, it has to be examined that these two interfere: components in a bad state will cause more track geometrical degradation, because they are more irregular then components in a good state and thus cause the train more to vibrate, causing a higher dynamic load; a vicious circle. This relation is also valid the other way: bad track geometry will in the same negative way affect the state of the track material. The relationships are visible in the figure 1. 


\section{Maintenance and renewal}

The choice for the maintenance or renewal action to be carried out and the appropriate time to do this is the subject of a long-time maintenance and renewal policy optimisation process. But in principle the maintenance or renewal are complementary, you ca even continue until the end of ages with replacing only single components (which is more of a maintenance action) and every time welding new pieces on worn metal parts, without replacement. Maintenance and renewal are even related in another way: it is quite useless to replace a worn rail with a new rail on a track which is in a bad geometrical state. Similar inefficient is improving the track geometry, while at the same time a worn rail might provoke such high dynamic forces on the track construction that the proper geometry will not last long. Also the component choice might be well placed if relative to the track geometry demanded (no heavy rail on a tight curve as normal on a tram line) and related to other components in place. These relationships are expressed in the figure 2 .

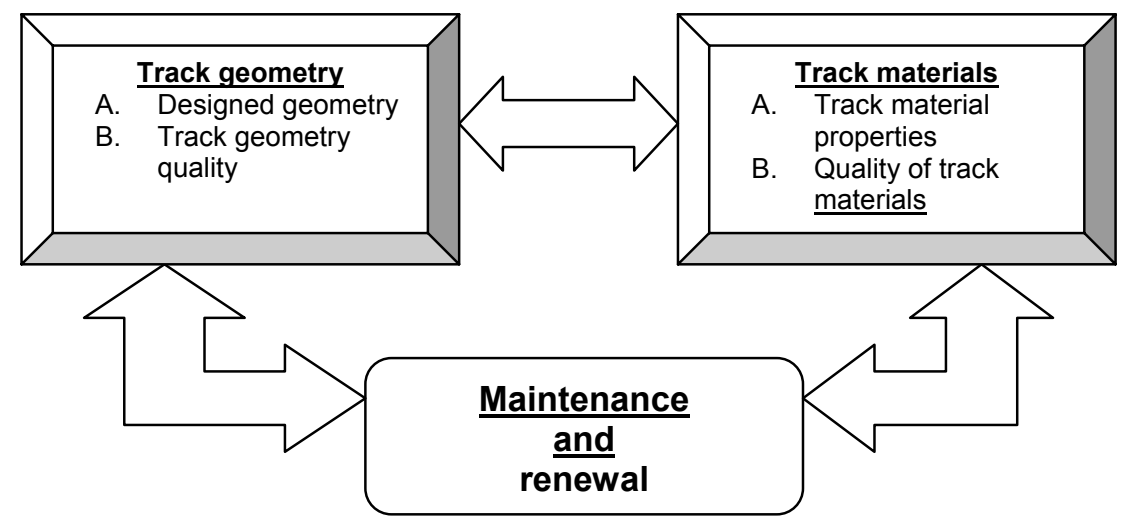

Figure 2: Relationships between the (state of the) track materials and track geometry (quality) and the maintenance and renewal actions.

\section{Methodology}

Combining figure 1 and 2 provides support for the following conclusions:

The track geometry degradation function depends on

- $\quad$ The speed, type and number of trains

- The actual track geometrical state

- The actual track components state

The track component wear and tear function depends on

- The speed, type and number of trains

- The actual track geometrical state

- The actual track components state 
It is therefore necessary, for an analyses or estimation of the life expectancy of a switch or crossing (or one of its component), to know the history of all these factors in detail to obtain a reliable degradation prediction. Unfortunately this is not the case; the data stored on the actual condition of S\&C is not available in a digital format (regular site inspections will only reveal if a component failed are satisfied a test, but not how much the actual wear was).

For this study therefore another analyses has been carried out. This has to do with the effect of different parameters on the expected life of S\&C. In this case standard turnouts (ST) on all Swiss main tracks with a cumulative daily load of more than 30.000 tons are analyzed.

The parameters taken into account are:

- the fact if a switch is curved or not

- $\quad$ switch angle

- $\quad$ soil quality

Standard turnout

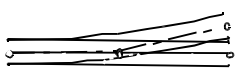

Diamond crossing

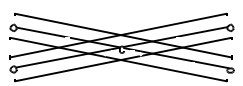

Symmetrical turnout

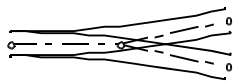

Diamond crossing with single slip

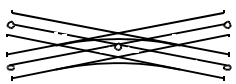

Combined turnouts

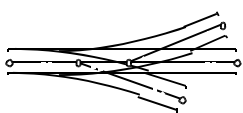

Diamond crossing with double slips

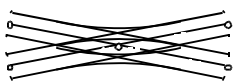

Figure 3: $\quad$ Types of switches and crossings.

\section{Results}

A way to express the effect of different parameters on the life expectancy is by drawing the estimated life time distribution curves. On the y axis it is shown which percentage is replaced after an x-amount of years or tonnage (depending on what is expressed on the $\mathrm{x}$-axis). An example is given in figure 4 .

\subsection{Life time general distribution}

The life time available in the databases is registered in years and causes big steps in an estimated lifetime distribution $(F)$ analyses. The tonnage is derived from the time-registration and calculated by taking half the tonnage from the year of installation and the year of replacement and the complete tonnage for the years in between. This simplification could be made because during winter time less switches and crossings are replaced, leading to more replacements during the summertime, which is approximately the middle of the year.

In figure 4 is visible that the maximum lifetime of standard turnouts is approximately equal to 30 years and 600 million tons. As a comparison, a 60 

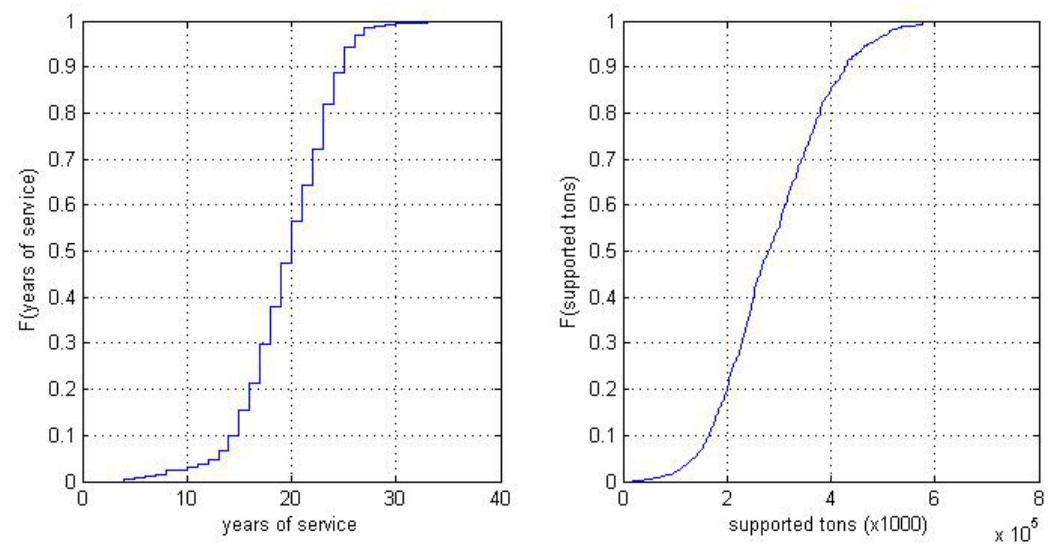

Figure 4: Estimated lifetime distribution functions of standard turnout replacements, left in years, right in tons.

kilogram per meter rail (UIC 60, 60E1) as installed on new tracks and on existing tracks subjected to heavy loads will normally also last 600 million tons. However, before reaching that age, all the $\mathrm{S} \& \mathrm{C}$ are nowadays already replaced.

Also visible is the effect of the steps in years, which is smoothed by recalculating towards tonnages. Because of the importance of tonnages, confirmed by experts, all the following graphs will present tonnages.

\subsection{Curved turnouts versus straight turnouts}

A general opinion among railway experts is that curved switches are more expensive than straight ones, because they are subjected to higher loads and more difficult to position correctly. This might lead to a shorter expected life time.

From figure 5 can be concluded that, at least for this dataset, no significant difference could be found for the distribution of expected life duration of curved or straight turnout. However this same test should be repeated for the maintenance and renewal needs for the components on curved and straight turnout to be able to state something on the maintenance and renewal needs. It also needs a check with experts if the non-registered maintenance works are perhaps different for curved turnouts, leading it to be in a slightly better state and thus resulting in the same life expectation as a straight turnout.

\subsection{Influence of the switch angle}

S\&C with a large angle and a tight curve with a small radius (1:7 to $1: 10)$ are very often subjected to large dynamic forces. This can be noticed just before a train arrives at a platform, through large sideway accelerations of the trains. These accelerations are provoked by the small curve in a switch or crossing and reflected back to it, because of the inertia of the train. 


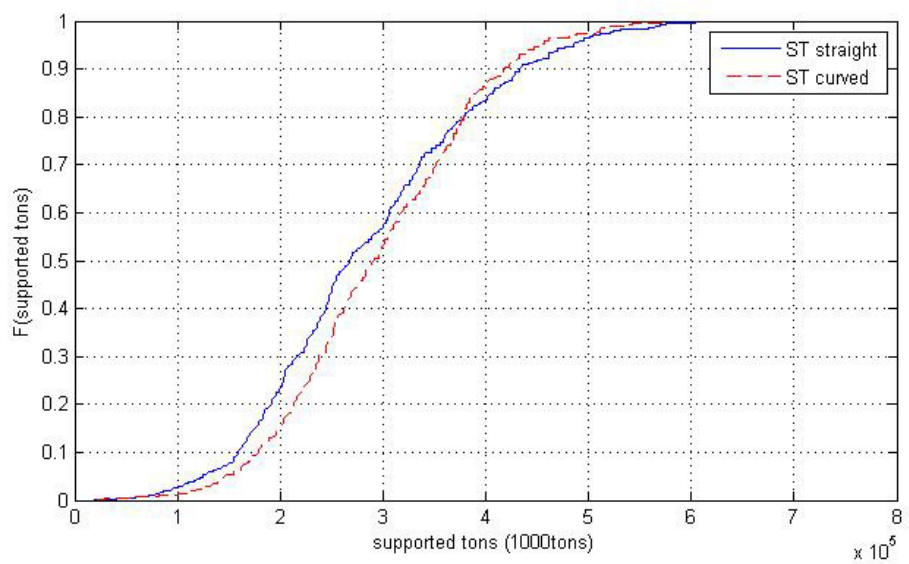

Figure 5: Estimated lifetime distribution functions of curved and straight standard turnouts (ST).

So this would lead to more wear and thus perhaps even a faster replacement need. This is more or less what figure 6 describes.

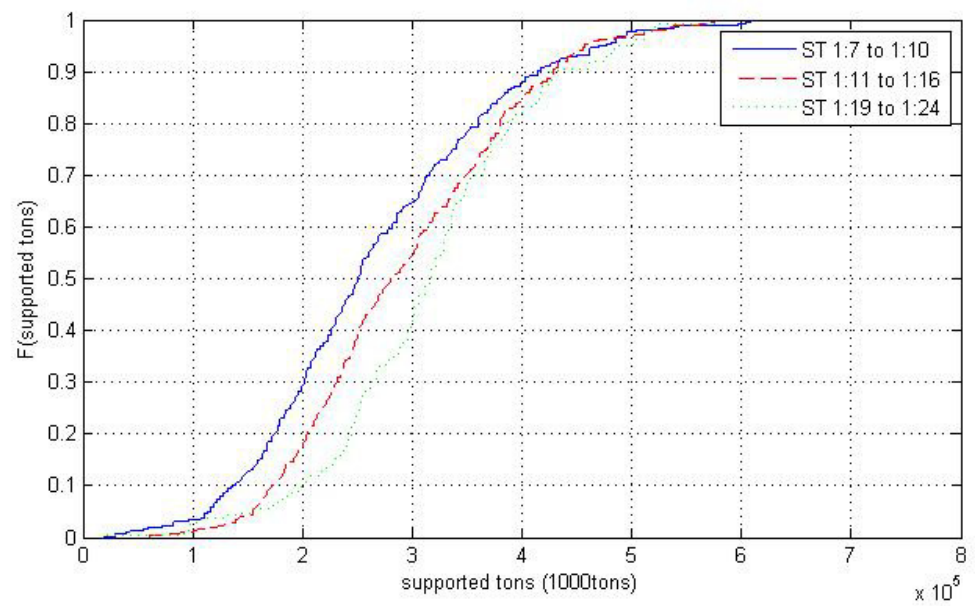

Figure 6: $\quad$ Estimated lifetime distribution functions of standard turnouts (ST) with different angles.

It can be seen in the figure above that standard turnouts with a small angle (1:19 to $1: 24)$ last longer in the track at least for the first part of the figure. At the same time it might also be noticed that the $\mathrm{S} \& \mathrm{C}$ with a small angle are always used in both directions with high speeds and with high daily tonnages, because they are expensive devices and they are mainly installed for capacity and 
timetable reasons (e.g. where a main line splits). It is thus also clear that these $\mathrm{S} \& \mathrm{C}$ are well maintained and are quickly subjected to support high tonnages. This on the contrary to the $\mathrm{S} \& \mathrm{C}$ with big angles and tight curves, who are often in places where speeds are slow, or when they are almost only used in one direction by almost all the trains; e.g. when providing an access to an industrial yard with only 1 train per week. This difference can through this analysis be introduced in the general degradation model as a difference between the switch angles regarding the expected renewal moment.

\subsection{Influence of the soil quality}

The soil quality is estimated to have a big impact on the life of a switch or crossing. Bad soil, will supply only heterogeneous support and thus cause higher dynamic loads.

In Switzerland 3 soil categories are distinguished: 1 for very good and 3 for very bad. For approximately 1800 switches and crossings, on highly loaded tracks, it was possible to determine the soil quality. The results are presented in figure 7.

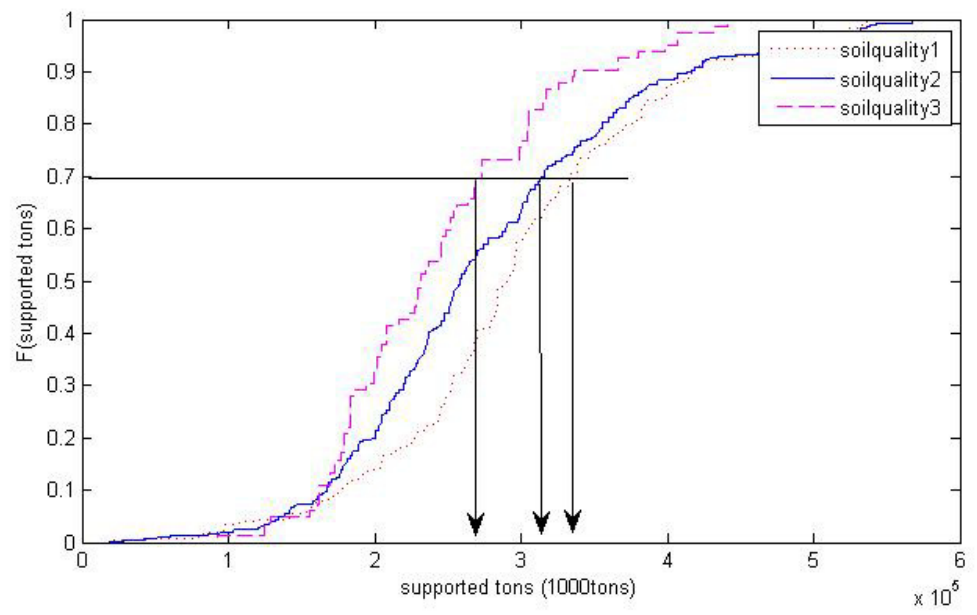

Figure 7: $\quad$ Estimated lifetime distribution functions of standard turnouts (ST) on different soil quality.

It can be clearly seen that $70 \%$ of the standard turnouts is already replaced after a cumulative tonnage of approximately 260 million tons in case of (bad) soil quality 3 , but the same percentage for (good) soil quality 3 is only reached after 340 million tons.

\section{Conclusion}

What can be concluded from the data in this database is that some parameters have a bigger effect than other, on the amount of time a switch can stay in place, 
or how much tons it can support. From big to small (or negligible): soil quality, switch angle and if it is curved or not.

However the database should be regarded with prudence. There are a lot of standard turnouts according to this dataset replaced far before their designed life expectancy in as well tons as years. This might have easy explanations like noise in the database, integral renewal (a turnout is renewed together with the track, even when not necessary, but for example because the track was built with another rail profile), infrastructure improvements or abandonment of infrastructure and thus the switch connecting this abandoned infrastructure to the main line.

An option for this problem would be to introduce truncation on the dataset, which means that switches and crossings with a life time less than a certain threshold are treated separately and not together with the big dataset on which the model is based.

Further research should also be conducted to explain the large range in as well time as tons of the life expectancy as presented in the life expectancy. The next step in this study is to devise a multiparametric model, which takes into account all parameters at the same time.

\section{Acknowledgement}

The author would like to acknowledge the Swiss Federal Railways (SBB CFF FFS) for supplying the data for this study.

\section{References}

[1] Jovanović, S. \& Zwanenburg, W.-J. Switches and Crossings Management System: EcoSwitch - Feasibility Study, ERRI Project Report D251/RP1 European Rail Research Institute, Utrecht, The Netherlands, 2002

[2] Rivier, R.E. Maintenance d'ouvrages et d'infrastructures, Lecture notes "Gestion de la maintenance des réseaux ferroviaires" EPFL - LITEP, Lausanne, Switzerland 2002

[3] Veit, P. Outsourcing of Track Maintenance, Presentation EFRTC General Assembly, slide 8-1, 2005 